\title{
Effects of Neuromuscular Electrical Stimulation for Masseter Muscle on Oral Dysfunction After Stroke
}

\author{
Kyeong Woo Lee, $\mathrm{MD}^{1,2}$, Sang Beom Kim, $\mathrm{MD}^{1,2}$, Jong Hwa Lee, $\mathrm{MD}^{1,2}$, \\ Sook Joung Lee, $\mathrm{MD}^{1,2}$, Jin Gee Park, $\mathrm{MD}^{1,2}$, Kyung Won Jang, $\mathrm{MD}^{1,2}$ \\ ${ }^{1}$ Department of Physical Medicine and Rehabilitation, Dong-A University College of Medicine, Busan; \\ ${ }^{2}$ Regional Cardiocerebrovascular Center, Dong-A Medical Center, Busan, Korea
}

Objective To determine positive effect of neuromuscular electrical stimulation (NMES) in conventional dysphagia therapy on masseter muscle oral dysfunction of patients after subacute stroke.

Methods Among subacute stroke patients who were diagnosed as oropharyngeal dysphagia by videofluoroscopy swallowing study (VFSS), those with oral dysfunction were enrolled. They were randomly assigned to a study group or a control group. The study group received NMES on masseter muscle and suprahyoid muscle simultaneously, while the control group received NMES only on suprahyoid muscle. NMES therapy session as applied 30 minutes each time, two times per day for a total of 20 sessions. Both groups received conventional dysphagia therapy for 2 weeks. All enrolled patients were evaluated by VFSS after 2 weeks. Oropharyngeal swallowing function was evaluated by Penetration-Aspiration Scale, Functional Dysphagia Scale (FDS), and American Speech-LanguageHearing Association National Outcome Measurement System swallowing scale based on results of VFSS.

Results Patients were randomly assigned to the study group $(n=20)$ or the control group $(n=20)$. There were no significant differences in baseline characteristics or initial values between the two groups. After 2 weeks of NMES, both groups showed improvement in scores of total FDS and pharyngeal phase FDS. Additionally, the study group showed improvement in oral phase FDS. Changes in all measurements were similar between the two groups.

Conclusion In this preliminary study, NMES for masseter muscle has a therapeutic effect on oral dysfunction of patients after subacute stroke.

Keywords Stroke, Post-stroke dysphagia, Oral phase dysfunction, Videofluoroscopy swallowing study, Neuromuscular electrical stimulation, NMES, Masseter muscle

Received July 27, 2018; Accepted October 10, 2018

Corresponding author: Kyung Won Jang

Department of Physical Medicine and Rehabilitation, Dong-A Medical Center, 26 Daesingongwon-ro, Seo-gu, Busan 49201, Korea. Tel: +82-51-2405690, Fax: +82-51-254-8511, E-mail: wkdruddnjs94@naver.com

ORCID: Kyeong Woo Lee (http://orcid.org/0000-0001-8785-5535); Sang Beom Kim (http://orcid.org/0000-0002-5622-5933); Jong Hwa Lee (http:// orcid.org/0000-0003-2489-358X); Sook Joung Lee (http://orcid.org/0000-0002-6894-445X); Jin Gee Park (http://orcid.org/0000-0003-2672-1618); Kyung Won Jang (http://orcid.org/0000-0001-8024-722X).

(c) This is an open-access article distributed under the terms of the Creative Commons Attribution Non-Commercial License (http://creativecommons.org/ licenses/by-nc/4.0) which permits unrestricted noncommercial use, distribution, and reproduction in any medium, provided the original work is properly cited. Copyright @ 2019 by Korean Academy of Rehabilitation Medicine 


\section{INTRODUCTION}

Stroke patients are susceptible to numerous complications. Among these potential complications, post-stroke dysphagia is common and important, with incidence ranging from $25 \%$ to $85 \%$. It is associated with mortality, increased pulmonary complications, and decreased quality of life [1-3]. The term 'dysphagia' refers to difficulty swallowing. The act of swallowing is a very complicated, multilevel, and neuromuscularly integrated event. It consists of three phases based on the location of the bolus, namely oral, pharyngeal, and esophageal phases $[4,5]$. Various dysphagia symptoms can exist in each phase. Thus, it is important to identify an appropriate treatment method for each swallowing symptom. For this reason, the effectiveness of various available treatment techniques and exercises has previously been studied according to each specific symptom of dysphagia. However, most recent researches on post-stroke dysphagia have been focused on pharyngeal phase dysfunction because subglottic aspiration occurs in the pharyngeal phase that may result in aspiration pneumonia, a critical and common pulmonary complication [6]. Nonetheless, oral phase dysfunction is as significant as pharyngeal phase dysfunction in patients. The oral phase is the first stage of swallowing. Oral phase dysfunctions such as inadequate bolus formation, oral transit time delay, and large amounts of residue in the oral cavity after swallowing may occur, resulting in insufficient intake of food that may trigger malnutrition or dehydration [7-9].

It is important to recognize that treatment for poststroke dysphagia involves both compensatory and restorative approaches [10]. Recently, several adjunctive treatment options have been reported, including surface neuromuscular electrical stimulation, pharyngeal electrical stimulation, repetitive transcranial magnetic stimulation, and transcranial direct current stimulation [11]. They can potentially improve patient's recovery from dysphagia. Of these treatment options, neuromuscular electrical stimulation (NMES) could both facilitate muscle activation and provide sensory stimulation. Most NMES studies have applied NMES to the laryngeal muscle of post-stroke dysphagia patients, thereby preventing subglottic aspiration through laryngeal elevation. These prior studies have investigated NMES therapy as a treatment modality for pharyngeal dysphagia in stroke pa- tients [12-14]. However, only a few studies have examined the effect of NMES on oral phase dysphagia following a stroke. Therefore, we hypothesized that NMES applied to the masseter muscle known to play an important role in mastication in the first stage of the swallowing process might be efficient to prevent oral phase dysfunction by improving chewing efficacy through motor strengthening effect and sensory stimulation. Thus, the aim of this study was to investigate the effect of NMES for masseter muscle on oral dysfunction after subacute stroke patients.

\section{MATERIALS AND METHODS}

\section{Subjects}

This study was designed as a pilot randomized controlled trial. Subacute stroke patients who were referred to the Regional Cardiocerebrovascular Center at our hospital between June 2015 and December 2017 were reviewed. Among patients who were diagnosed as oropharyngeal dysphagia by videofluoroscopy swallowing study (VFSS), those with oral dysfunction according to the Functional Dysphasia Scale (FDS) were then included in this study [15]. Oral dysfunction included any abnormality of lip closure, bolus formation, residue in oral cavity, and oral transit time that resulted in an oral dysfunction diagnosis.

Of patients who were initially diagnosed with oral dysfunction, those with a past history of stroke, those with an oral cavity anatomical abnormality, those who were unable to cooperate due to decreased mental capacity or severely deteriorated cognitive function caused by stroke (Mini-Mental State Examination score $<10$ ), and those who exhibited a poor medical condition that could affect their swallowing ability were excluded from this study.

\section{Methods}

\section{NMES therapy}

Enrolled patients were randomly assigned to one of the two groups. The study group received NMES (VitalStim, Chattanooga group, Hixson, TN, USA) for masseter muscle and suprahyoid muscle simultaneously. The control group received NMES only for the suprahyoid muscle (Fig. 1). NMES was applied in biphasic waveform at frequency of $80 \mathrm{~Hz}$ with phase duration of $300 \mathrm{~ms}$.

The amplitude of the electrical current level was approximately $7 \mathrm{~mA}$. Clinician confirmed appropriate 


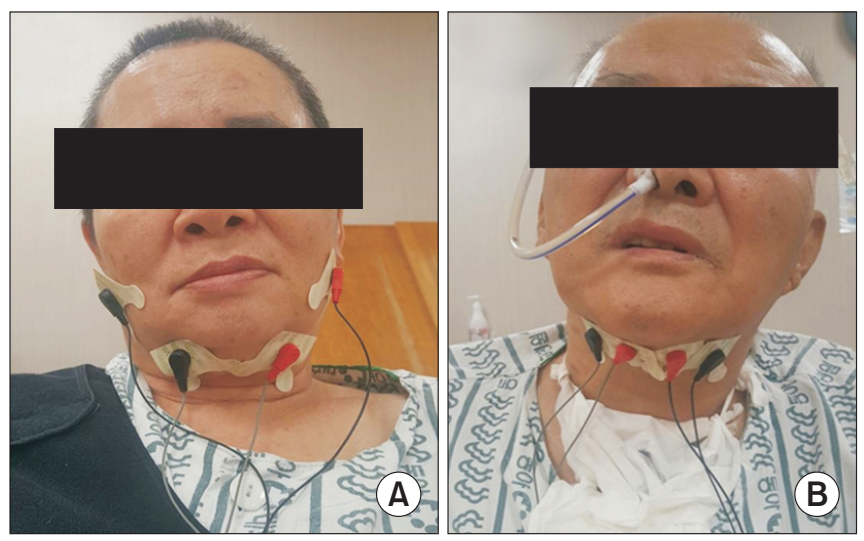

Fig. 1. (A) Study group receiving NMES for masseter muscle and suprahyoid muscle. (B) Control group receiving NMES for suprahyoid muscle. NMES, neuromuscular electrical stimulation.

electrical stimulation on masseter muscle by inspection and palpation of masseter muscle. NMES therapy session was applied 20 minutes each time, two times per day for a total of 20 sessions. Both groups received conventional dysphagia therapy comprising oral motor and sensory stimulation with oral and lingual exercises that focused on strength and endurance to improve the efficacy and safety of the swallowing process [16]. Ten sessions of conventional swallowing rehabilitation therapy were conducted for both study and control groups once daily, 5 days a week for 2 weeks, with each session lasting 30 minutes.

\section{Assessment}

To evaluate the swallowing ability of patients in the two groups before and after the 2-week rehabilitation training, American Speech-Language-Hearing Association National Outcome Measurement System swallowing scale (ASHA-NOMS), FDS, and Penetration-Aspiration Scale (PAS) were used. VFSS was performed for patients in sitting position to allow lateral view. A modified version of the protocol from a study performed by Logemann was used [17]. The ASHA-NOMS criteria comprise 1-8 stages depending on patient's dietary pattern and the range of possible meals. 'Stage 1' indicates the most severe condition of dysphagia while 'Stage 8' indicates the mildest condition. It is correlated with the severity of dysphagia [18].

FDS is a scale developed to quantify dysphagia severity. It consists of 11 items with weighted values repre- senting four kinds of oral (lip closure, bolus formation, residues in oral cavity, oral transit time) and seven kinds of pharyngeal (triggering of pharyngeal swallow, laryngeal elevation and epiglottic closure, nasal penetration, residue in valleculae, residue in pyriformis sinus, coating of pharyngeal wall after swallow, pharyngeal transit time) functions. The maximum possible score is 100. It is achieved by applying different weight values to each item. Thus, it is useful for quantifying the degree of swallowing difficulty in dysphagia patients and quantitatively determine the effectiveness of dysphagia treatment [18]. In relation to oral functions in FDS, scores can range from 0 to 28 points. When evaluating lip closure, intact, inadequate, and no lip closures are scored as 0,5 , and 10 points, respectively. Bolus formation is evaluated according to the following: absence of residual food, score of 0 ; $<10 \%$ of residual food, score of 2 points; $10 \%-50 \%$ of residual food, score of 4 points; and $>50 \%$ of residual food, score of 6 points. The severity of residue in the oral cavity is scored as $0,2,4$, or 6 points when there is no residue, $<10 \%$ residue, $10 \%-50 \%$ residue, or $>50 \%$ residue, respectively. The presence of an oral transit time delay of 1.5 seconds is scored as 0 while the absence of such a delay is scored 6 points for evaluation.

The PAS evaluates airway invasions. It has a maximum score of 8 points. Scores are determined primarily based on the depth to which material passes into the airway, whether material passes below the vocal fold, and where there is any effort to eject the material. A PAS score of 8 means that material enters the airway and passes below vocal folds without effort made to eject it. It is represented as silent aspiration caused by weak or absence of reflex cough [19]. All test procedures were recorded and findings were analyzed by two physiatrists.

Basic information including age, sex, type of stroke, and the time from diagnosis to VFSS evaluation of stroke patients were investigated. The study protocol was approved by the Institutional Review Board at Dong-A Medical Center (No. DAUH IRB-18-147). All participants were given a written informed consent form, and they signed the consent form prior to the enrollment.

\section{Statistical analysis}

All statistical analyses were performed with SPSS version 18.0 (SPSS Inc., Chicago, IL, USA). Differences in demographic characteristics and parameters of swallow- 
ing function between the two groups at baseline were analyzed using the Mann-Whitney test. Statistical significance in results between before and after treatment was determined using the Wilcoxon signed-rank test. To investigate differences in swallowing function between the two groups, we performed the Mann-Whitney test. A p-value less than 0.05 was considered statistically significant.

\section{RESULTS}

A total of 57 subacute stroke patients with oral phase dysfunction were initially enrolled. However, 14 patients with a past history of pulmonary disease, neurological damage, or medical complications that could affect Velopharyngeal Insufficiency (VPI) were unable to cooperate. Thus, they were excluded. The remaining 43 patients were randomized and divided into the study group and the control group for treatment. Three subjects who dropped out because of early discharge, recurrent stroke, or deterioration of medical condition were excluded from this study. Finally, 40 patients ( 20 in the study group and
20 in the control group) were enrolled. The study group included 8 men and 12 women with an average age of $66.24 \pm 15.62$ years and the control group included 10 men and 10 women with an average age of $64.65 \pm 12.83$ years. In the study group, 10 patients had cerebral infarction and 10 had cerebral hemorrhage. The average time from stroke onset to their first VFSS was $16.35 \pm 10.24$ days. In the control group, 12 patients had cerebral infarction and $8 \mathrm{had}$ cerebral hemorrhage. The average time from stroke onset to the first VFSS was $18.96 \pm 8.94$ days. In the study group, the swallowing score of the ASHA-NOMS was stage $3.83 \pm 1.45$ and FDS was $7.65 \pm 3.25$ points for the oral phase, $1.83 \pm 1.87$ points for residues in the oral cavity, $5.64 \pm 1.55$ points for oral transit time, $25.85 \pm 8.75$ points for the pharyngeal phase, $33.46 \pm 13.44$ points in total, and $6.75 \pm 1.21$ points for the PAS. In the control group, the swallowing score of the ASHA-NOMS was stage $3.63 \pm 1.64$ and FDS was $7.82 \pm 4.12$ points for the oral phase, $1.78 \pm 1.95$ points for residues in oral cavity, $5.94 \pm 1.89$ points for oral transit time, $26.83 \pm 8.69$ points for the pharyngeal phase, $34.75 \pm 11.46$ points in total, and $6.62 \pm 1.53$ points for PAS. Baseline evaluation of the two

Table 1. Baseline characteristics of subjects at initial evaluation

\begin{tabular}{|lccc}
\hline & Study group $(\mathbf{n = 2 0})$ & Control group $(\mathbf{n = 2 0})$ & p-value \\
\hline Sex & & & 1.000 \\
\hline Male & $8(40.0)$ & $10(50.0)$ & \\
\hline Female & $12(60.0)$ & $10(50.0)$ & \\
\hline Cause of dysphagia & & & \\
\hline Hemorrhage & $10(50.0)$ & $12(60.0)$ & \\
\hline Infarction & $10(50.0)$ & $8(40.0)$ & 0.764 \\
\hline Days from onset to therapy & $16.35 \pm 10.24$ & $18.96 \pm 8.94$ & 0.842 \\
\hline Age (yr) & $66.24 \pm 15.62$ & $64.65 \pm 12.83$ & 0.348 \\
\hline MMSE & $19.27 \pm 7.24$ & $20.71 \pm 8.18$ & 0.728 \\
\hline ASHA-NOMS & $3.83 \pm 1.45$ & $3.63 \pm 1.64$ & 0.379 \\
\hline Oral phase FDS (0-28) & $7.65 \pm 3.38$ & $7.82 \pm 3.12$ & \\
\hline Lip closure (0-10) & 0 & 0 & 0.652 \\
\hline Bolus formation (0-6) & 0 & $1.78 \pm 1.95$ & 0.485 \\
\hline Residues in oral cavity (0-6) & $1.83 \pm 1.87$ & $5.94 \pm 1.89$ & 0.884 \\
\hline Oral transit time (0-6) & $5.64 \pm 1.55$ & $26.83 \pm 8.69$ & 0.695 \\
\hline Pharyngeal FDS (0-72) & $25.85 \pm 8.75$ & $34.75 \pm 11.46$ & 0.452 \\
\hline Total FDS (0-100) & $33.46 \pm 13.44$ & $6.62 \pm 1.53$ & \\
\hline PAS (1-8) & $6.75 \pm 1.21$ & & \\
\hline
\end{tabular}

Values are presented as mean \pm standard deviation.

MMSE, Mini-Mental State Examination; ASHA-NOMS, American Speech-Language-Hearing Association National Outcome Measurement System swallowing scale; FDS, Functional Dysphagia Scale; PAS, Penetration-Aspiration Scale. 
groups showed no significant difference (Table 1).

After 2 weeks of rehabilitation, the study group showed significant improvements in swallowing scores of FDS in the oral phase, the pharyngeal phase, and total (Table 2). In contrast, no statistically significant differences were observed in ASHA-NOMS or PAS (Table 2). In the control group, significant improvements were observed in swallowing scores of FDS in the pharyngeal phase and total (Table 2). In contrast, no statistically significant differences were observed in ASHA-NOMS, FDS in the oral phase, or FDS in PAS (Table 2). A comparison of the therapeutic effect between the two groups showed no statistically significant difference in the swallowing score for ASHA-
NOMS, FDS in the oral phase, FDS in the pharyngeal phase, FDS in total, or FDS in PAS (Table 3).

\section{DISCUSSION}

This study aimed to investigate effects of NMES therapy in subacute stroke patients with oral phase. Results revealed that the study group showed significant improvement in their oral phase dysfunction according to FDS compared to the control group. A previous study has reported that application of NMES for masticatory muscles can improve muscle activity in elderly chronic stroke patients [20]. However, the report did not demon-

Table 2. Changes of swallowing and cough functions

\begin{tabular}{|lccccccc}
\hline & \multicolumn{3}{c}{ Study group (n=20) } & & \multicolumn{3}{c}{ Control group (n=20) } \\
\cline { 2 - 3 } \cline { 7 - 8 } & Pre & Post (2 weeks) & p-value & & Pre & Post (2 weeks) & p-value \\
\hline ASHA-NOMS (1-7) & $3.83 \pm 1.45$ & $4.75 \pm 1.58$ & 0.061 & & $3.63 \pm 1.64$ & $4.58 \pm 1.63$ & 0.063 \\
Oral phase FDS (0-28) & $7.65 \pm 3.25$ & $2.35 \pm 2.43$ & $0.041^{*}$ & & $7.82 \pm 4.12$ & $4.10 \pm 3.58$ & 0.074 \\
\hline Lip closure (0-10) & 0 & 0 & & & 0 & 0 & \\
\hline Bolus formation (0-6) & 0 & 0 & & & 0 & 0 & \\
\hline Residues in oral cavity (0-6) & $1.83 \pm 1.87$ & $0.95 \pm 0.87$ & 0.138 & & $1.78 \pm 1.95$ & $1.05 \pm 0.95$ & 0.167 \\
\hline Oral transit time (0-6) & $5.64 \pm 1.55$ & $1.54 \pm 1.34$ & 0.076 & & $5.94 \pm 1.89$ & $3.12 \pm 2.42$ & 0.158 \\
\hline Pharyngeal FDS (0-72) & $25.85 \pm 8.75$ & $17.66 \pm 5.71$ & $0.039^{*}$ & & $26.83 \pm 8.69$ & $20.22 \pm 9.22$ & $0.036^{*}$ \\
\hline Total FDS (0-100) & $33.46 \pm 13.44$ & $20.36 \pm 9.45$ & $0.046^{*}$ & & $34.75 \pm 11.46$ & $24.32 \pm 11.27$ & $0.038^{*}$ \\
\hline PAS (1-8) & $6.75 \pm 1.21$ & $5.34 \pm 0.78$ & 0.068 & $6.62 \pm 1.53$ & $5.24 \pm 1.35$ & 0.087 \\
\hline
\end{tabular}

Values are presented as mean \pm standard deviation.

ASHA-NOMS, American Speech-Language-Hearing Association National Outcome Measurement System swallowing scale; FDS, Functional Dysphagia Scale; PAS, Penetration-Aspiration Scale.

${ }^{*} \mathrm{p}<0.05$ by Wilcoxon signed-rank test.

Table 3. Comparison of changes between the study group and the control group

\begin{tabular}{|lccc}
\hline & Study group $(\mathbf{n = 2 0})$ & Control group $(\mathbf{n}=\mathbf{2 0})$ & p-value $^{\text {a) }}$ \\
\hline ASHA-NOMS (1-7) & $0.92 \pm 0.53$ & $0.95 \pm 0.64$ & 0.630 \\
\hline Oral phase FDS (0-28) & $-5.30 \pm 1.75$ & $-3.72 \pm 2.35$ & 0.122 \\
\hline Lip closure (0-10) & 0 & 0 & \\
\hline Bolus formation (0-6) & 0 & 0 & 0.262 \\
\hline Residues in oral cavity (0-6) & $-0.87 \pm 0.65$ & $-0.72 \pm 0.72$ & 0.183 \\
\hline Oral transit time (0-6) & $-4.52 \pm 0.89$ & $-2.68 \pm 1.59$ & 0.317 \\
\hline Pharyngeal FDS (0-72) & $-8.22 \pm 4.67$ & $-6.65 \pm 4.42$ & 0.252 \\
\hline Total FDS (0-100) & $-13.12 \pm 3.81$ & $-10.55 \pm 3.57$ & 0.326 \\
\hline PAS (1-8) & $-1.41 \pm 1.83$ & $-1.38 \pm 1.50$ & \\
\hline
\end{tabular}

Values are presented as mean \pm standard deviation.

ASHA-NOMS, American Speech-Language-Hearing Association National Outcome Measurement System swallowing scale; FDS, Functional Dysphagia Scale; PAS, Penetration-Aspiration Scale.

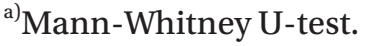


strate changes of swallowing function after NMES. Thus, this study investigated changes of swallowing function through NMES for masseter muscle in subacute stroke patients with oral phase dysfunction. Patients with oral phase dysfunction may fail to swallow sufficient food, particularly when the amount of residue in the oral cavity is increased or when oral transit time is delayed. As a result, patients may undergo malnutrition and dehydration. When tubal feeding is applied in patients to prevent malnutrition and dehydration, problems such as gastroesophageal reflux disease, agitation, discomfort, and difficulty with sleeping can occur. Additionally, prolonged complications such as inflammation, tissue fibrosis, and adhesion resulting from sustained exposure of the nasal cavity or esophagus to a rubber foreign body may arise [21-23].

Few studies have been conducted on oral phase dysfunction. One study has found that delayed oral transit is related to low cognitive function which is more likely when stroke lesion is in the left frontal lobe. Nevertheless, strokes occur in different locations. Therefore, it is not easy to predict oral phase dysphagia only with imaging of lesions [24]. In addition, Stroke patients manifest different clinical symptoms. Oral phase dysfunction could present in various stroke lesions [25]. There are currently multiple treatment options available for post-stroke dysphagia. Among these treatment options, NMES uses electrical impulses to stimulate muscles and elicit muscle contraction or mimic normal automatic contraction, thereby improving or recovering the function of stimulated muscles.

Multiple studies have verified the efficacy of NMES. Therefore, NMES is widely used in the clinical field [12]. In a clinical setting, NMES is usually applied to the infrahyoid or suprahyoid muscles to achieve laryngeal elevation during the pharyngeal phase in order to prevent subglottic aspiration $[13,14]$. In addition to muscular strengthening effect, such electrical impulses may also provide sensory stimulation. It has been shown that transcutaneous electrical stimulation to treat sensory impairment in post-stroke dysphagia can improve cortical sensory motor circuits [26]. A previous study has reported that NMES applied to the peripheral nerve can influence functional measures of motor performance in subjects with stroke and can additionally produce changes in cortical excitability [27]. In another study, change of cor- tical activation pattern has occurred with improvement of motor function in stroke patients throughout NMES [28]. Furthermore, decreased orofacial motor function in stroke patients has been shown, resulting in oral phase dysfunction. The swallowing process is a sequential coordination of oropharyngeal muscles, not just a series of separate swallowing processes. Thus, if mastication is not effective, it can contribute to oral phase dysfunction [29]. A recent study has also shown that oral phase dysfunction is closely associated with the initial mastication process [30]. Thus, we hypothesized that the application of NMES to the masseter muscle could enhance the chewing process during the oral phase by cortical excitability and that such improvement in the oral phase might facilitate the entire swallowing process in post-stroke dysphagia patients. Our results revealed that oral phase dysfunction characterized by orofacial motor and sensory impairments could be improved by applying NMES to the masseter muscle. In addition, the study group showed improved pharyngeal phase and overall FDS scores compared to the control group, indicating that enhanced oral phase function might contribute to overall improvement in the swallowing process. These changes demonstrate that NMES for masseter muscle can influence swallowing function in subjects with stroke. Such improvement could be due to changes in cortical excitability throughout NMES.

This study is important because research on treatment is limited for oral phase dysfunction. Moreover, existing treatments could be used in new ways. For example, NMES could be applied to the masseter muscle rather than to the laryngeal elevator muscle alone. However, further studies are needed to compare differences in long-term therapeutic effects and the incidence of oral phase dysfunction. Moreover, oral dysfunction was defined by using subscores of FDS. Therefore, other types such as oral apraxia were excluded. Another limitation of this study was that patients with severely deteriorated cognitive function were excluded. In addition, those with oral dysfunction arising from aphasia, apathy, or depression were also excluded. Furthermore, the comparison in this study was performed with a relatively small number of patients. Thus, further studies with larger sample sizes that consider direct chewing function should be conducted in the future to validate results of the present study. In conclusion, this preliminary study found that appli- 
cation of NMES to the masseter muscle had a therapeutic effect on oral dysfunction of patients after subacute stroke. Hence, NMES of the masseter muscle could represent a viable treatment option for oral dysfunction after stroke. Additionally, chewing might play an important role in stimulating the initiation of the swallowing process. Thus, NMES of the masseter muscle could also enhance the chewing process. Improvement of the oral phase might facilitate the swallowing process as a whole. However, future studies with a larger number of participants focusing on the oral phase are necessary to confirm findings of this study.

\section{CONFLICT OF INTEREST}

No potential conflict of interest relevant to this article was reported.

\section{REFERENCES}

1. Martino R, Foley N, Bhogal S, Diamant N, Speechley M, Teasell R. Dysphagia after stroke: incidence, diagnosis, and pulmonary complications. Stroke 2005;36: 2756-63.

2. Gonzalez-Fernandez M, Ottenstein L, Atanelov L, Christian AB. Dysphagia after stroke: an overview. Curr Phys Med Rehabil Rep 2013;1:187-96.

3. Huang KL, Liu TY, Huang YC, Leong CP, Lin WC, Pong YP. Functional outcome in acute stroke patients with oropharyngeal Dysphagia after swallowing therapy. J Stroke Cerebrovasc Dis 2014;23:2547-53.

4. Bosma JF. Deglutition: pharyngeal stage. Physiol Rev 1957;37:275-300.

5. Dodds WJ, Stewart ET, Logemann JA. Physiology and radiology of the normal oral and pharyngeal phases of swallowing. AJR Am J Roentgenol 1990;154:953-63.

6. Armstrong JR, Mosher BD. Aspiration pneumonia after stroke: intervention and prevention. Neurohospitalist 2011;1:85-93.

7. Broadley S, Croser D, Cottrell J, Creevy M, Teo E, Yiu D, et al. Predictors of prolonged dysphagia following acute stroke. J Clin Neurosci 2003;10:300-5.

8. Finestone HM, Greene-Finestone LS, Wilson ES, Teasell RW. Malnutrition in stroke patients on the rehabilitation service and at follow-up: prevalence and predictors. Arch Phys Med Rehabil 1995;76:310-6.
9. Gordon C, Hewer RL, Wade DT. Dysphagia in acute stroke. Br Med J (Clin Res Ed) 1987;295:411-4.

10. Hughes T. Management of adult neurogenic dysphagia. Brain 1999;122:1604-5.

11. Geeganage C, Beavan J, Ellender S, Bath PM. Interventions for dysphagia and nutritional support in acute and subacute stroke. Cochrane Database Syst Rev 2012;10:CD000323.

12. Chen YW, Chang KH, Chen HC, Liang WM, Wang YH, Lin YN. The effects of surface neuromuscular electrical stimulation on post-stroke dysphagia: a systemic review and meta-analysis. Clin Rehabil 2016;30:24-35.

13. Kiger M, Brown CS, Watkins L. Dysphagia management: an analysis of patient outcomes using VitalStim therapy compared to traditional swallow therapy. Dysphagia 2006;21:243-53.

14. Park JW, Oh JC, Lee HJ, Park SJ, Yoon TS, Kwon BS. Effortful swallowing training coupled with electrical stimulation leads to an increase in hyoid elevation during swallowing. Dysphagia 2009;24:296-301.

15. Han TR, Paik NJ, Park JW. Quantifying swallowing function after stroke: a functional dysphagia scale based on videofluoroscopic studies. Arch Phys Med Rehabil 2001;82:677-82.

16. Vose A, Nonnenmacher J, Singer ML, Gonzalez-Fernandez M. Dysphagia management in acute and subacute stroke. Curr Phys Med Rehabil Rep 2014;2:197206.

17. Palmer JB, Kuhlemeier KV, Tippett DC, Lynch C. A protocol for the videofluorographic swallowing study. Dysphagia 1993;8:209-14.

18. Sallum RA, Duarte AF, Cecconello I. Analytic review of dysphagia scales. Arq Bras Cir Dig 2012;25:279-82.

19. Rosenbek JC, Robbins JA, Roecker EB, Coyle JL, Wood JL. A penetration-aspiration scale. Dysphagia 1996; 11:93-8.

20. Wang JS, Lee JH, Kim NJ. Effects of neuromuscular electrical stimulation on masticatory muscles in elderly stroke patients. J Phys Ther Sci 2015;27:2767-70.

21. Alivizatos V, Gavala V, Alexopoulos P, Apostolopoulos A, Bajrucevic S. Feeding tube-related complications and problems in patients receiving long-term home enteral nutrition. Indian J Palliat Care 2012;18:31-3.

22. Metheny NA, Meert KL, Clouse RE. Complications related to feeding tube placement. Curr Opin Gastroenterol 2007;23:178-82. 
23. Rowat A. Enteral tube feeding for dysphagic stroke patients. Br J Nurs 2015;24:138-45.

24. Moon HI, Yoon SY, Yi TI, Jeong YJ, Cho TH. Lesions responsible for delayed oral transit time in post-stroke dysphagia. Dysphagia 2018;33:321-8.

25. Daniels SK, Pathak S, Mukhi SV, Stach CB, Morgan RO, Anderson JA. The relationship between lesion localization and dysphagia in acute stroke. Dysphagia 2017;32:777-84.

26. Gallas S, Marie JP, Leroi AM, Verin E. Sensory transcutaneous electrical stimulation improves post-stroke dysphagic patients. Dysphagia 2010;25:291-7.

27. Kimberley TJ, Lewis SM, Auerbach EJ, Dorsey LL, Lojovich JM, Carey JR. Electrical stimulation driving functional improvements and cortical changes in subjects with stroke. Exp Brain Res 2004;154:450-60.

28. Shin HK, Cho SH, Jeon HS, Lee YH, Song JC, Jang SH, et al. Cortical effect and functional recovery by the electromyography-triggered neuromuscular stimulation in chronic stroke patients. Neurosci Lett 2008;442: 174-9.

29. Schimmel M, Voegeli G, Duvernay E, Leemann B, Muller F. Oral tactile sensitivity and masticatory performance are impaired in stroke patients. J Oral Rehabil 2017;44:163-71.

30. Palmer JB, Rudin NJ, Lara G, Crompton AW. Coordination of mastication and swallowing. Dysphagia 1992;7:187-200. 